Pacific Journal of Mathematics

ABELIAN GROUPS WHICH ADMIT ONLY NILPOTENT 


\title{
ABELIAN GROUPS WHICH ADMIT ONLY NILPOTENT MULTIPLICATIONS
}

\section{WILLIAM J. WICKLESS}

\begin{abstract}
Let $\langle G+\rangle$ be an abelian group. A ring $R$ with additive group $\langle R+\rangle$ isomorphic to $\langle G+\rangle$ is a ring on $G . G$ is nil (radical) if and only if $R^{2}=(0)(R$ is nilpotent) for all rings $R$ on $G$. It is shown that $G$ is a mixed radical group if and only if $T$ is divisible and $G / T$ is radical, where $T$ is the maximal torsion subgroup of $G$. Thus, the study of radical groups is reduced to the torsion free case. A torsion free group $G$ is of field type if and only if there exists a ring $R$ on $G$ such that $Q \otimes R$ is a field. It is shown that a torsion free group of finite rank is radical if and only if it has no strongly indecomposable component of field type. It follows that finite direct sums of finite rank radical groups are radical. If $G$ is torsion free an element $x \in G$ is of nil type if and only if the height vector $h(x)=\left\langle m_{\imath}\right\rangle$ is such that $0<m_{i}<\infty$ for infinitely many $i$. Multiplications on torsion free groups all of whose nonzero elements are of nil type are discussed under the assumption of three chain conditions on the partially ordered set of types. Two special classes of rank two torsion free radical groups are characterized. An example is given of a torsion free radical group homogeneous of non-nil type, and a simple condition is given for such a homogeneous group to be nonradical.
\end{abstract}

1. Radical groups-the torsion and mixed case. Several authors ([4], [7], [8], [9]) have studied abalian groups which admit only a trivial ring structure. These are called nil groups.

We wish to consider a larger class of abelian groups-those abelian groups which admit only a nilpotent ring structure. More precisely:

DEFINITION 1.1. Let $\langle G+\rangle$ be an abelian group. A ring $R$ with additive group $\langle R+\rangle$ isomorphic to $\langle G+\rangle$ is called a ring on $G$.

Definition 1.2. An abelian group $G$ is a radical group if and only if whenever $R$ is a ring on $G$ we have $R^{k}=(0)$ for some positive integer $k$.

Hereafter we use the word group to mean abelian group. In [4] it is shown that a torsion group is nil if and only if it is divisible. The same theorem and proof hold with "nil" replaced by "radical", so in the torsion case radical and nil groups coincide. 
For mixed groups we have the following simple result.

THEOREM 1.1. Let $G$ be a mixed group with maximal torsion subgroup $T$. Then $G$ is radical if and only if $T$ is divisible and $G / T$ is radical.

Proof. Let $G$ be a mixed radical group with maximal torsion subgroup T. $T$ must be divisible, for otherwise $G$ would have a cyclic direct summand and would not be a radical group. Since $T$ is divisible we have $G=T \oplus F$, and clearly $F$ must also be a radical group.

Conversely, let $G$ be a mixed group such that $T$ is divisible and $G / T$ is radical. Assume $G$ is (isomorphic to) the additive group of some ring. Since $T$ is torsion divisible we have $T^{2}=(0)$; and because $T$ is an ideal in any ring on $G$, the given multiplication on $G$ induces a multiplication on $G / T$. Since $G / T$ is radical we have $(G / T)^{k}=(0)$ and thus $G^{2 k}=(0)$. Hence, $G$ is radical.

2. Torsion free radical groups of finite rank. It is clear from $\S 1$ that the study of radical groups can be reduced to the torsion free case.

In this section we use the results of [1] and [5] to obtain a necessary and sufficient condition that a torsion free group of finite rank be radical and to obtain some information about direct sums of radical groups.

First we recall some definitions and results from [1] and [5].

Definition 2.1. Let $A$ and $B$ be groups. $A$ is quasi-isomorphic to $B(A \sim B)$ if and only if there exist subgroups $A^{\prime} \subseteq A, B^{\prime} \subseteq B$ such that $A^{\prime} \cong B^{\prime}$ and $A / A^{\prime}$ and $B / B^{\prime}$ are of bounded order.

In [5] it is shown that if $A$ is a torsion free group of finite rank, we have $A \sim A_{1} \oplus \cdots \oplus A_{l}$ with each $A_{i}$ strongly indecomposable in the sense that $A_{i}$ is not quasi-isomorphic to a direct sum of two nonzero torsion free groups. This is called a quasi-decomposition of $A$ and the $A_{i}$ are called the strongly indecomposable components of the decomposition. Moreover, it is shown that if $A \sim$ $A_{1} \oplus \cdots \oplus A_{l}$ and $A \sim B_{1} \oplus \cdots \oplus B_{n}$ are two quasi-decompositions of $A$ into strongly indecomposable components, then $l=n$ and with suitable rearrangement, $A_{i} \sim B_{i}$ for all $i$.

Definition 2.2. Let $G$ be a torsion free group. $G$ is of field type if and only if there exists a ring $R$ on $G$ with $Q \otimes R$ a field. Here $Q$ is the field of rational numbers, the tensor product is taken 
over the integers, and the ring multiplication on $R$ is extended to $Q \otimes R$ in the obvious way.

In [1] it is shown that if $R$ is a torsion free ring of finite rank, then $R \sim M_{1} \oplus \cdots \oplus M_{k} \oplus N$ where each $M_{i}$ is a group of field type and $N$ is the maximal nilpotent ideal of $R$. (If $R$ is nilpotent, then $R=N$ and no $M_{i}$ appears.)

Lemma 2.1. Let $A$ and $B$ be torsion free groups with $A \sim B$. $A$ is a radical group (is of field type) if and only if $B$ is a radical group (is of field type).

Proof. This is a special case of Corollary 2.7 of [1] and is easy to verify directly.

The following theorem was suggested by R. S. Pierce.

Theorem 2.1. Let $A$ be a torsion free group of finite rank. $A$ is radical if and only if $A$ has no strongly indecomposable component of field type. (By our earlier remarks this will not depend on the particular quasi-decomposition of $A$ chosen.)

Proof. Let $A$ be a torsion free group of finite rank which is not radical. Then there exists a non-nilpotent multiplication on $A$. Thus, we have $A \sim M_{1} \oplus \cdots \oplus M_{k} \oplus N$, where $N$ is the maximal nilpotent ideal of $A$ and each $M_{i}$ is of field type. Since $A$ is not nilpotent at least one $M_{i}$ occurs.

Now consider $M_{1} \neq(0)$ as a torsion free ring for which $Q \otimes M_{1}$ is a field. Applying Corollary 5 of [6], we have $M_{1} \sim M_{1} \oplus \cdots \oplus$ $M_{\uparrow}^{l_{1}}$, where each $M_{\imath}^{2}$ is a strongly indecomposable torsion free group of field type.

For $i \leqq 2$ we write each $M_{2} \sim M_{\imath}^{\prime} \oplus \cdots \oplus M_{i}^{l_{i}}$ and $N \sim N_{1} \oplus \cdots \oplus$ $N_{t}$ where the $M_{i}^{\prime}$ and the $N_{i}$ are strongly indecomposable components of the groups $M_{i}$ and $N$. We note that $A \sim \sum_{i=1}^{k} \sum_{j_{i-1}}^{l_{i}} \oplus M_{1}^{j} \oplus$ $\sum_{1}^{t} \oplus N_{i}$ is a quasi-decomposition of $A$ into strongly indecomposable components for which each of the groups $M_{i}, \cdots, M_{1}^{l_{1}}$ is of field type.

The converse implication follows immediately from Lemma 2.1.

Corollary. Let $A_{1}, \cdots, A_{k}$ be torsion free radical groups of finite rank. Then $A_{1} \oplus \cdots \oplus A_{k}$ is radical.

We note that the corollary is false for countable direct sums of rank one torsion free nil groups. For a counterexample, choose a sequence of subgroups of the additive rationals $A_{1} \subset A_{2} \subset \cdots \subset A_{k} \subset$ $\cdots$ such that $H\left(A_{k}\right)=[\langle k, k, \cdots, k, \cdots\rangle]$. Each $A_{k}$ is torsion free 
nil of rank one. Let $R=\left\{a_{1} x+a_{2} x^{2}+\cdots+a_{n} x^{n} \mid a_{i} \in A_{i}\right\}$. We make $R$ into a ring via the usual polynomial operations. Then $R$ is a ring on $\sum_{i=1}^{\infty} \oplus A_{i}$ which is a Jacobson semisimple ring.

As in [4], if $A$ is any torsion free group with $a \in A$ and $p_{1}, \cdots$, $p_{h}, \cdots$ is the sequence of prime integers in their natural ordering, we let $h(a)=\left\langle m_{k}\right\rangle$ with $0 \leqq m_{k} \leqq \omega$ for all $k$ each $m_{k}$ being defined by the condition $p_{k}^{m}{ }_{k} X=a$ is solvable in $A$, but $p_{k}^{m / k} X=a$ is not solvable in $A$. The sequence $\left\langle m_{k}\right\rangle$ is called the height vector of $a$ in $A$.

We define an equivalence relation on the set $\{h(a) \mid a \in A\}$ by $h(a)=\left\langle m_{k}\right\rangle \sim\left\langle n_{k}\right\rangle=h(b)$ if and only if $m_{k}=n_{k}$ for almost all $k$ and if $m_{k} \neq n_{k}$ both $m_{k}$ and $n_{k}$ are finite. We let $H(a)$ be the equivalence class $[h(\alpha)]$. We say $H(a)$ is nil if and only if $H(\alpha)=\left[\left\langle m_{k}\right\rangle\right]$ with $0<m_{k}<\infty$ for infinitely many $k$.

We call a group $G$ homogeneous if and only if $H(a)=H(b)$ for any two nonzero elements $a, b \in G$. We set $H(G)=H(a)$, where $a$ is any nonzero element of $G$, and call $H(G)$ the type of $G$. It is easy to prove that if $G$ is a homogeneous torsion free group and $E$ is a torsion free group with $E \sim G$, then $E$ is homogeneous and $H(E)=$ $H(G)$.

THeorem 2.2. Let $A$ be a torsion free group of finite rank with no strongly indecomposable component homogeneous of non nil type. Then $A$ is radical.

Proof. We combine Theorem 2.1 and the observation that if $M$ is the additive group of a ring with $Q \otimes M$ a field, then $M$ is homogeneous of non nil type.

COROLLARY. For an arbitrary torsion free group $G$ the following conditions are equivalent:

(1) $H(x)$ is nil for all nonzero $x \in G$.

(2) All finite rank pure subgroups of $G$ are radical.

Proof. $1 \rightarrow 2$ is immediate from Theorem 2.2 and the definition of purity. $2 \rightarrow 1$ since $H(x)$ is just the type of the rank one pure subgroup of $G$ generated by $x$, and it is well known that a rank one torsion free group is radical (nil) if and only if it is of nil type.

We close this section with an interesting observation due to Pierce.

THEOREM 2.3. If $A$ is a strongly indecomposable torsion free group of finite rank and $A$ admits one nilpotent nonzero multiplication, then $A$ is radical. 
Proof. Let $A$ be a group satisfying the above conditions. Suppose $A$ is not radical. Then $A$ admits a multiplication of field type. Applying Corollary 4.6 of [2], we have $Q \otimes \operatorname{Hom}(A, A)$ is an algebraic number field. But if $A$ admits a nilpotent nonzero multiplication, then $\operatorname{Hom}(A, A)$ has nonzero nilpotent elements--a contradiction.

3. Torsion free groups all of whose elements are of nil type.

Definition 3.1. Let $G$ be a torsion free group. We say all of the elements of $G$ are of nil type if and only if $x \in G, x \neq 0$ implies $H(x)$ is nil.

The example following Theorem 2.1 shows that a group $G$ with all elements of nil type need not be radical. If such $G$ is also of finite rank, then by the Corollary to Theorem 2.2, it is radical.

As in [4], if $a, b \in A, A$ a torsion free group, we say $H(a) \leqq H(b)$ if we can write $H(a)=\left[\left\langle m_{k}\right\rangle\right], H(b)=\left[\left\langle n_{k}\right\rangle\right]$ with $m_{k} \leqq n_{k}$ for all $k$. This defines a partial ordering on the set $H=\{H(a) \mid a \in A\}$. It is well known that if $A$ is of finite rank, then $H$ satisfies the ascending and descending chain conditions.

In this section we study multiplications on groups with all elements of nil type, under the assumptions of three chain conditions on $H$. We prove a lemma which will be useful throughout.

LEMma 3.1 Let $A$ be a torsion free ring with elements $a, b$ of nil type. Then $H(a b)>H(a)$ or $H(a b)>H(b)$.

Proof. Clearly $H(a b) \geqq H(a)$ and $H(a b) \geqq H(b)$. Let $h(a)=\left\langle m_{t}\right\rangle$, $h(b)=\left\langle n_{t}\right\rangle, h(a b)=\left\langle l_{t}\right\rangle$. If $H(a b)=H(a)$, then $l_{t}=m_{t}$ for almost all $t$. Since $l_{t} \geqq m_{t}+n_{t}$, then $n_{t}=0$ for almost all $t$ such that $l_{t}<$. So for all $t$, we have $l_{t} \geqq n_{t}$, and for the infinitely many $t$ with $0<l_{t}<c$, we have $0=n_{t}<l_{t}$. Thus $H(a b)>H(b)$.

THEOREM 3.1. Let $G$ be a torsion free group with all elements of nil type and let $H$ be the set of types of elements of $G$. Assume that the lengths of all strictly ascending chains in $H$ are bounded by some fixed positive integer $n$. Then $G$ is radical.

Proof. Let $G$ be as above and assume that $G$ is the additive group of some ring. For all positive integers $k$, let $V_{k}=\left\{x \in G \mid \exists_{y_{2}}\right.$, $\cdots, y_{k}$ such that $\left.H(x)>H\left(y_{2}\right)>\cdots>H\left(y_{k}\right)\right\}$, and let $G_{k}$ be the subgroup of $G$ generated by the elements of $V_{k}$. 
We have $G=G_{1} \supseteqq G_{2} \supseteqq \cdots \supseteqq G_{k} \supseteqq \cdots$ is a descending sequence of subgroups of $G$, and by Lemma 3.1 we note that $G_{k}^{2} \subseteq G_{k+1}$ for all $k$. Our hypothesis says that $G_{n+1}=(0)$. The result follows.

THEOREM 3.2. Let $G$ be as in Theorem 3.1 and $H$ the set of types of elements of $G$. Assume that $H$ satisfies the ascending chain condition. Then every ring on $G$ is a Baer radical ring. (Divinsky, § 3.3.)

Proof. Suppose we have (defined a multiplication on $G$ as above for which $\beta(G) \neq G$, where $\beta(G)$ denotes the Baer radical of $G$. Let $T$ be the set theoretic complement of $\beta(G)$ in $G$ and $V=\{y \in T \mid H(y)$ is maximal in $T\}$. Let $I=\left\{x+\beta(G) \mid x+\beta(G)=\sum_{i=1}^{l} y_{i}+\beta(G), y_{i} \in V\right\}$. It is easy to check that $I$ is a nonzero ideal in the ring $G / \beta(G)$ and that $I^{2}=(0)$. (Lemma 3.1) This is a contradiction, so we must have $\beta(G)=G$ for all multiplications on $G$.

THEOREM 3.3. Let $G$ be a torsion free group with all elements of nil type and $H$ be its set of types. Assume that for all $x \in G$ there exist elements $x_{1}, \cdots, x_{l}$ of $G$ and positive integers $n_{1}, \cdots, n_{l}$ such that $x=x_{1}+\cdots+x_{l}$ and the length of any strictly ascending chain in $H$ starting with $H\left(x_{i}\right)$ is bounded by $n_{i}, i=1 \cdots l$. Then any ring on $G$ is a nil ring.

Proof. Let $G$ be as above. For all positive integers $n$, let $V_{n}=$ $\left\{x \in G \mid \exists\right.$ no $y_{2}, \cdots, y_{n} \in G$ such that $\left.H(x)<H\left(y_{2}\right)<\cdots<H\left(y_{n}\right)\right\}$, and let $G_{n}$ be the subgroup of $G$ generated by the elements of $V_{n}$. We have $(0)=G_{1} \subseteq G_{2} \subseteq \cdots \sqsubseteq G_{n} \subseteq \cdots$ is an ascending chain of subgroups of $G$. Applying Lemma 3.1, we have for any $\operatorname{ring}$ on $G G_{n}^{2} \subseteq$ $G_{n-1}$ for all $n \geqq 2$. The assumption of our theorem says that $U_{n=1}^{\infty}$ $G_{n}=G$. The result follows.

4. Rank two torsion free radical groups. In this section we use the rank one groups defined in [3] to give a simple characterization of two classes of rank two torsion free radical groups.

We recall some definitions and results from [3]. Let $G$ be a torsion free rank two group with independent elements $x$ and $y$. Let $A=\{\alpha \in Q \mid \alpha x+\beta y \in G$ for some $\beta \in Q\}, A_{0}=\{\alpha \in Q \mid \alpha x \in G\}, B=\{\beta \in$ $Q \mid \alpha x+\beta y \in G$ for some $\alpha \in Q\}, B_{0}=\{\beta \in Q \mid \beta y \in G\}$. The rank one groups $A, A_{0} ; B, B_{0}$ are called the groups belonging to $x$ and $y$ respectively. Clearly $A_{0} \cong A, B_{0} \subseteq B$. It is shown in [2] that $A / A_{0} \cong B / B_{0}$ and that any torsion free group of rank two is essentially determined by $A, A_{0}, B, B_{0}$ and the isomorphism between $A / A_{0}$ and $B / B_{0}$. Also if 
$A_{0} \subseteq A, B_{0} \subseteq B$ are rank one torsion free groups with an isomorphism $\theta: A / A_{0} \rightarrow B / B_{0}$, then $G=\left\{(\alpha, \beta) \mid \alpha \in A, B_{0}=\theta\left(\alpha+A_{0}\right)\right\}$ is a torsion free group of rank two with componentwise addition having independent elements $x, y$ such that $A, A_{0} ; B, B_{0}$ are the groups belonging to $x$ and $y$.

THEOREM 4.1. A torsion group $G$ of rank two is radical and not nil if and only if there exist independent elements $x, y \in G$ such that if $A, A_{0} ; B, B_{0}$ are the groups belonging to $x$ and $y$, we have

(1) $A, B, B_{0}$ are nil

(2) $A_{0} \leqq A \leqq B_{0} \subseteq B$

(3) $H\left(B_{0}\right) \geqq 2 H(A)$.

Proof. Suppose that $G$ is a radical but not nil torsion free group of rank two. Then there exists a multiplication of $G$ such that $G^{3}=$ (0) but $G^{2} \neq(0)$. It is easy to prove directly (see [1]) that for such a ring on $G$ we can find $x \in G$ such that $x$ and $x^{2}$ are rationally independent. Let $A, A_{0}$ and $B, B_{0}$ be the rank one groups belonging to $x$ and $x^{2}$ respectively. Let $\alpha \in A$. Then $\alpha x+\beta x^{2} \in G$ for some $\beta \in$ $Q$, whence $\alpha x^{2} \in G$ so $\alpha \in B_{0}$. Thus, we have $A_{0} \subseteq A \subseteq B_{0} \subseteq B$. Also if $\alpha x+\beta x^{2} \in G$ then $\left(\alpha x+\beta x^{2}\right)^{2} \in G$, so for all $\alpha \in A$ we have $\alpha^{2} \in B_{0}$. This yields $H\left(B_{0}\right) \geqq 2 H(A)$.

Finally, we show $A, B_{0}, B$ are nil groups. Let $\left\langle k_{i}\right\rangle=h_{A}(1),\left\langle k_{i}^{0}\right\rangle=$ $h_{A_{0}}(1),\left\langle l_{i}\right\rangle=h_{B}(1),\left\langle l_{i}^{0}\right\rangle=h_{B_{0}}(1)$ be the height vectors of the integer 1 in the four groups as indicated by the subscripts. Because of the containment relation we have $k_{i}^{0} \leqq k_{i} \leqq l_{i}^{0} \leqq l_{i}$. Since $A / A_{0} \cong B / B_{0}$ it is easy to check that $k_{i}-k_{i}^{0}=l_{i}-l_{i}^{0}$ for all $i$, with the convention $\infty-\infty=0, \infty-n=\infty$ for $n$ finite. We note that $H(A)$ must be nil, for otherwise $k_{i}=0$ or $k_{i}=\infty$ for all but a finite number of $i$. This would imply that $k_{i}=k_{i}^{0}, l_{i}=l_{i}^{0}$ for all but a finite number of $i$, and when $k_{i} \neq k_{i}^{0}, l_{i} \neq l_{1}^{0}$ all are finite. But then $G \sim A \oplus B$ and $A$ is not a nil (radical) group-a contradiction. Similar arguments show that $B_{0}$ and $B$ are nil.

Conversely, let $G$ be a torsion free group of rank two with independent elements $x, y$ and groups $A, A_{0} ; B, B_{0}$ belonging to $x$ and $y$ satisfying Conditions $1-3$ of our theorem. For any ring on $G$, suppose $y^{2}=u x+v y$. Then for all $\beta \in B_{0},(\beta y)^{2}=\beta^{2} u x+\beta^{2} v y$, so $\beta^{2} u \in A$-a contradiction unless $u=0$ since $H\left(B_{0}\right) \geqq 2 H(A)$. Thus $y^{2}=$ $v y$. But $B_{0}$ is nil, so $y^{2}=v y$ only if $v=0$. Thus $y^{2}=0$.

Now let $x y=r x+t y$. For all $\beta \in B_{0}$ we have $x(\beta y)=r \beta x+t \beta y$, so $r \beta \in A$. This is impossible unless $r=0$, because $B_{0}$ and $A$ are of nil type and $H\left(B_{0}\right) \geqq 2 H(A)$. Thus $x y=t y$. Similarly, $y x=l y$.

Finally, if $x^{2}=\gamma x+\delta y$, then for all $\alpha \in A \alpha x+\beta y \in G$ for some 
$\beta$ and $(\alpha x+\beta y)^{2}=\alpha^{2} \gamma x+s y$. Thus for all $\alpha \in A$ we have $\alpha^{2} \gamma \in A$ a contradiction unless $\gamma=0$ since $A$ is nil. Hence $x^{2}=\delta y$. We see that any multiplication on $G$ must be nilpotent, so $G$ is radical. Using $A_{0} \subseteq A \subseteq B_{0} \subseteq B$ and $H\left(B_{0}\right) \geqq 2 H(A)$ we can define a non-zero multiplication on $G$ by specifying the products $y^{2}=x y=y x=0, x^{2}=$ $m y$ for $m$ chosen in the obvious way. This extends consistently to all of $G$. Thus $G$ is not nil.

Definition 4.1. Let $G$ be a torsion free group. $G$ is a strong radical group if and only if all torsion free homomorphic images of $G$ are radical.

THEOREM 4.2. Let $G$ be a torsion free group of rank two. $G$ is a strong radical group if and only if for all rationally independent elements $x, y \in G$ and rank one groups $A, A_{0} ; B, B_{0}$ belonging to $x$ and $y$, we have $A$ and $B$ are of nil type.

Proof. Let $G$ be rank two strong radical with $x, y$ rationally independent. Let $A, A_{0} ; B, B_{0}$ be the groups belonging to $x$ and $y$. We note that $A^{*}$ and $B$ are homomorphic images of $G$ via the homomorphisms $\theta$ and $\varphi$ given by $\theta(\alpha x+\beta y)=\alpha, \varphi(\alpha x+\beta y)=\beta$. Thus, $A$ and $B$ are rank one radical. Thus $H(A), H(B)$ are nil.

Conversely, let $G$ be torsion free of rank two and not strong radical. Then there exists an onto homomorphism $\theta: G \rightarrow T$, where $T$ is torsion free and not radical. If rank $T=1$, choose $0 \neq t \in T$ and $g \in G$ with $\theta(g)=t$. Choose $0 \neq n \in$ ker $\theta$. Then $\langle g, n\rangle$ is a maximal independent set in $G$ with rank one groups $A, A_{0} ; B, B_{0}$ such that $A \cong T$. We have $H(A)=H(T)$ is not nil.

If $\operatorname{rank} T=2, G \cong T$, and we can assume $G$ is not radical. Since $G$ is rank two and not radical, we have $G$ is of field type or $G \sim$ $U \oplus V$ where $U$ is a rank one group of field type. In the first case, Theorem 5 of [3] says that we can find independent elements $x, y$ such that $A$ and $B$ are not nil. In the second case, it is easy to construct a maximal independent set $\langle x, y\rangle$ in $G$ such that the rank one group $A$ belonging to $x$ is not nil. This proves the theorem.

5. Torsion free groups homogeneous of non-nil type. In this section we show that, even for groups of rank two, the converse to Theorem 2.2 is false. We give a simple theorem giving one case in which a homogeneous non-nil type group is not radical.

The following is an example of a torsion free rank two radical group which is strongly indecomposable and homogeneous of type $[\langle 0, \cdots, 0, \cdots\rangle]$. Let $s=s_{0}+s_{1} p+s_{2} p^{2}+\cdots$ be a $p$-adic unit which 
satisfies no polynomial with rational coefficients. Let $S_{k}=s_{0}+s_{1} p+\cdots$ $s_{k-1} p^{k-1} . \quad\left(S_{0}=0.\right) \quad$ Let $G=\left\{\left(r / p^{k}, r / p^{k} S_{k}+t\right) \mid r, t \in Z,(r, p)=1\right\} . \quad G$ is a rank two subgroup of $Q \oplus Q$. By using Corollary 5.27 and Theorem 8.6 of [1], it is not hard to show that $G$ is strongly indecomposable and radical. It is easy to check directly that $G$ is homogeneous of type $[\langle 0, \cdots, 0, \cdots\rangle]$.

If $G$ is an arbitrary torsion free group with maximal rationally independent set $\left\{x, y_{\alpha}, \alpha \in B\right\}$, let $A(x)=\left\{q \in Q \mid \exists q_{1} \cdots q_{n} \in Q, \alpha_{1} \cdots \alpha_{n} \in\right.$ $B$ with $\left.q x+q_{1} y_{\alpha_{1}}+\cdots+q_{n} y_{\alpha_{n}} \in G\right\}, A_{0}(x)=\{q \in Q \mid q x \in G\}$.

THEOREM 5.1. Let $G$ be a torsion free group, homogeneous of non nil type. Assume there exists a maximal rationally independent set $\left\{x, y_{\alpha}, \alpha \in B\right\}$ in $G$ such that $H[A(x)]=H\left[A_{0}(x)\right]$. Then $G$ is not radical.

Proof. Let $G, A, A_{0}$ be as above. Since $A_{0} \subseteq A$ and $H\left(A_{0}\right)=H(A)$, there exists a positive integer $m$ with $m A \subseteq A_{0}$. We can define a non-nilpotent multiplication on $G$ by

$$
g g^{\prime}=\left(q x+q_{1} y_{\alpha_{1}}+\cdots+q_{n} y_{\alpha_{n}}\right)\left(q^{\prime} x+q_{1}^{\prime} y_{\alpha_{1}}+\cdots+q_{n}^{\prime} y_{\alpha_{n}}\right)=m^{2} q q^{\prime} x .
$$

Acknowledgment. We wish to thank Professor R. S. Pierce for his many helpful suggestions and improvements.

\section{REFERENCES}

1. R. A. Beaumont and R. S. Pierce, Torsion free rings, Illinois J. Math., 5 (1) 61-98.

2. Subrings of algebraic number fields, Acta Sci. Math., 22, 202-216.

3. R. A. Beaumont and R. J. Wisner, Rings with additive group which is a torsion free group of rank two, Acta Sci. Math. Szeged, 20, 105-116.

4. L. Fuchs, Abelian groups, Pergamon Press, New York, 1960.

5. B. Jonsson, On direct decompositions of torsion free abelian groups, Math. Scand.,

7, 361-371.

6. R. S. Pierce, Subrings of simple algebras, Mich. Math. J., 7, 241-243.

7. R. Ree and R. J. Wisner, A note on torsion free nil groups, Amer. Math. Soc. Proc., 7, 6-8.

8. T. Szele, Gruppentheorische beziehungen bei gewissen ringkonstruktionen, Math. Z., 54, 168-180.

9. Z Zur theorie der zeroringe, Math. Ann. 121, 242-246.

Received December 21, 1970 and in revised form July 19, 1971. The preparation of this paper was supported in part by NSF Grant \#GP-23107.

UNIVERSity of CALIFORNIA, LoS ANGELES 



\section{PACIFIC JOURNAL OF MATHEMATICS}

\section{EDITORS}

H. SAMELSON

Stanford University

Stanford, California 94305

C. R. HOBBY

University of Washington

Seattle, Washington 98105
J. DugundJI

Department of Mathematics

University of Southern California

Los Angeles, California 90007

RICHARD ARENS

University of California

Los Angeles, California 90024

\section{ASSOCIATE EDITORS}

E. F. BeCKENBACH

B. H. NeumanN

F. WOLF

K. YosHIDA

\section{SUPPORTING INSTITUTIONS}

UNIVERSITY OF BRITISH COLUMBIA

CALIFORNIA INSTITUTE OF TECHNOLOGY

UNIVERSITY OF CALIFORNIA

MONTANA STATE UNIVERSITY

UNIVERSITY OF NEVADA

NEW MEXICO STATE UNIVERSITY

OREGON STATE UNIVERSITY

UNIVERSITY OF OREGON

OSARA UNIVERSITY
UNIVERSITY OF SOUTHERN CALIFORNIA STANFORD UNIVERSITY

UNIVERSITY OF TOKYO

UNIVERSITY OF UTAH

WASHINGTON STATE UNIVERSITY

UNIVERSITY OF WASHINGTON

AMERICAN MATHEMATICAL SOCIETY

NAVAL WEAPONS CENTER

Printed in Japan by International Academic Printing Co., Ltd., Tokyo, Japan 


\section{Pacific Journal of Mathematics}

\section{Vol. 40, No. $1 \quad$ September, 1972}

Alex Bacopoulos and Athanassios G. Kartsatos, On polynomials

approximating the solutions of nonlinear differential equations........

Monte Boisen and Max Dean Larsen, Prüfer and valuation rings with zero

divisors ..........................................

James J. Bowe, Neat homomorphisms

David W. Boyd and Hershy Kisilevsky, The Diophantine equation

$$
u(u+1)(u+2)(u+3)=v(v+1)(v+2) \ldots \ldots \ldots \ldots \ldots \ldots \ldots
$$

George Ulrich Brauer, Summability and Fourier analysis ...............

Robin B. S. Brooks, On removing coincidences of two maps when only one,

rather than both, of them may be deformed by a homotopy ............

Frank Castagna and Geert Caleb Ernst Prins, Every generalized Petersen

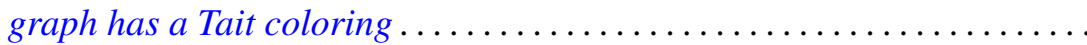

Micheal Neal Dyer, Rational homology and Whitehead products ..........

John Fuelberth and Mark Lawrence Teply, The singular submodule of a

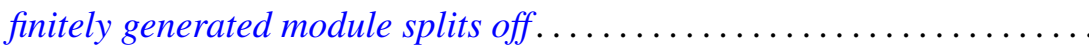

Robert Gold, $\Gamma$-extensions of imaginary quadratic fields ............ 83

Myron Goldberg and John W. Moon, Cycles in k-strong tournaments.......

Darald Joe Hartfiel and J. W. Spellmann, Diagonal similarity of irreducible

matrices to row stochastic matrices...............

Wayland M. Hubbart, Some results on blocks over local fields ..

Alan Loeb Kostinsky, Projective lattices and bounded homomorphisms....

Kenneth O. Leland, Maximum modulus theorems for algebras of operator

valued functions ...

Jerome Irving Malitz and William Nelson Reinhardt, Maximal models in the

language with quantifier "there exist uncountably many" ..

John Douglas Moore, Isometric immersions of space forms in space

forms.

Ronald C. Mullin and Ralph Gordon Stanton, A map-theoretic approach to

Davenport-Schinzel sequences ....................

Chull Park, On Fredholm transformations in Yeh-Wiener space. .

Stanley Poreda, Complex Chebyshev alterations ..............

Ray C. Shiflett, Extreme Markov operators and the orbits of Ryff. ...

Robert L. Snider, Lattices of radicals .....................

Ralph Richard Summerhill, Unknotting cones in the topological

category ................................

Charles Irvin Vinsonhaler, A note on two generalizations of $\mathrm{QF}-3 \ldots \ldots 229$

William Patterson Wardlaw, Defining relations for certain integrally

parameterized Chevalley groups...................

William Jennings Wickless, Abelian groups which admit only nilpotent

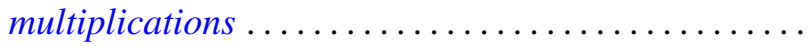

